Revue québécoise de droit international

Quebec Journal of International Law

Revista quebequense de derecho internacional

\title{
The Sound of Silence : le pouvoir discrétionnaire du Procureur de la Cour pénale internationale à travers l'utilisation des critères d'intérêts de la justice et de gravité lors de l'ouverture d'une enquête
}

\section{Claire Magnoux}

Numéro hors-série, décembre 2017

Études de certains grands enjeux de la justice internationale pénale

URI : https://id.erudit.org/iderudit/1056223ar

DOI : https://doi.org/10.7202/1056223ar

Aller au sommaire du numéro

Éditeur(s)

Société québécoise de droit international

ISSN

0828-9999 (imprimé)

2561-6994 (numérique)

Découvrir la revue

Citer cet article

Magnoux, C. (2017). The Sound of Silence : le pouvoir discrétionnaire du Procureur de la Cour pénale internationale à travers l'utilisation des critères d'intérêts de la justice et de gravité lors de l'ouverture d'une enquête. Revue québécoise de droit international / Quebec Journal of International Law / Revista quebequense de derecho internacional, 9-36. https://doi.org/10.7202/1056223ar
Résumé de l'article

Une des grandes innovations dans le domaine de la justice internationale pénale, à l'exception de l'avènement même d'une cour permanente, est la consécration, en son sein, d'un Procureur indépendant. Cette indépendance fait néanmoins l'objet d'un encadrement notamment à travers l'article $53 \mathrm{du}$ Statut de Rome de la Cour pénale internationale, intitulé « Ouverture d’une enquête ». En effet, celui-ci circonscrit l'action du Procureur dès la phase d'ouverture d'enquête (paragraphe 1) en établissant un certain nombre de critères, dont celui des " intérêts de la justice ". Ce critère, pensé comme un critère de pondération, permet au Procureur de refuser d'ouvrir une enquête même si l'ensemble des autres critères est présent. Il constitue ainsi le bastion du pouvoir discrétionnaire du Procureur. Dans la pratique, aucun refus d'ouverture d'enquête ne s'est jamais basé sur le critère des « intérêts de la justice ». Or, à l'exception des situations ne passant pas le test d'admissibilité, les refus d'ouverture d'enquête se sont basés sur le test de la recevabilité et précisément sur le critère de la gravité. Cela signifie-t-il que le Procureur restreint de lui-même son pouvoir discrétionnaire en se concentrant sur d'autres critères ? Notre démarche vise à démontrer que le processus d'objectivation auquel s'est livré le Procureur dans la prise en compte de ces deux critères (intérêts de la justice et gravité) a été nuisible à l'image d'impartialité voulue par ce dernier, nécessitant des ajustements de sa part. Ainsi, il a minimisé l'utilisation de l'application du critère des intérêts de la justice au profit de celui de la gravité à la faveur d'une interprétation stricte des « intérêts de la justice » (I), tout en prenant en compte ce critère par l'intermédiaire de stratégies détournées rendant le contrôle et les débats relatifs à ses choix plus compliqués (II). Or, nous pensons qu'au regard du type de conflictualité auquel il doit faire face, il est nécessaire de se questionner sur une évolution du spectre du critère des intérêts de la justice, et donc de sa prise en compte dans le choix d'ouverture d'une enquête (III).
Tous droits réservés @ Société québécoise de droit international, 2018
Ce document est protégé par la loi sur le droit d'auteur. L’utilisation des services d'Érudit (y compris la reproduction) est assujettie à sa politique d'utilisation que vous pouvez consulter en ligne.

https://apropos.erudit.org/fr/usagers/politique-dutilisation/ 


\title{
THE SOUND OF SILENCE : LE POUVOIR DISCRÉTIONNAIRE DU PROCUREUR DE LA COUR PÉNALE INTERNATIONALE À TRAVERS L'UTILISATION DES CRITÈRES D'INTÉRÊTS DE LA JUSTICE ET DE GRAVITÉ LORS DE L'OUVERTURE D'UNE ENQUÊTE
}

\author{
Claire Magnoux*
}

\begin{abstract}
Une des grandes innovations dans le domaine de la justice internationale pénale, à l'exception de l'avènement même d'une cour permanente, est la consécration, en son sein, d'un Procureur indépendant. Cette indépendance fait néanmoins l'objet d'un encadrement notamment à travers l'article 53 du Statut de Rome de la Cour pénale internationale, intitulé "Ouverture d'une enquête». En effet, celui-ci circonscrit l'action du Procureur dès la phase d'ouverture d'enquête (paragraphe 1) en établissant un certain nombre de critères, dont celui des «intérêts de la justice ». Ce critère, pensé comme un critère de pondération, permet au Procureur de refuser d'ouvrir une enquête même si l'ensemble des autres critères est présent. Il constitue ainsi le bastion du pouvoir discrétionnaire du Procureur. Dans la pratique, aucun refus d'ouverture d'enquête ne s'est jamais basé sur le critère des « intérêts de la justice ». Or, à l'exception des situations ne passant pas le test d'admissibilité, les refus d'ouverture d'enquête se sont basés sur le test de la recevabilité et précisément sur le critère de la gravité. Cela signifie-t-il que le Procureur restreint de lui-même son pouvoir discrétionnaire en se concentrant sur d'autres critères? Notre démarche vise à démontrer que le processus d'objectivation auquel s'est livré le Procureur dans la prise en compte de ces deux critères (intérêts de la justice et gravité) a été nuisible à l'image d'impartialité voulue par ce dernier, nécessitant des ajustements de sa part. Ainsi, il a minimisé l'utilisation de l'application du critère des intérêts de la justice au profit de celui de la gravité à la faveur d'une interprétation stricte des « intérêts de la justice» (I), tout en prenant en compte ce critère par l'intermédiaire de stratégies détournées rendant le contrôle et les débats relatifs à ses choix plus compliqués (II). Or, nous pensons qu'au regard du type de conflictualité auquel il doit faire face, il est nécessaire de se questionner sur une évolution du spectre du critère des intérêts de la justice, et donc de sa prise en compte dans le choix d'ouverture d'une enquête (III).
\end{abstract}

One of the main innovations in the field of international criminal law, except for the advent of a permanent court in and of itself, is the consecration, within this court, of an independent Prosecutor. This independence, however, is subject to a specific framework, namely through article 53 of the Rome Statute of the International Criminal Court, "Initiation of an investigation". Indeed, this article frames the action of the Prosecutor from the opening of an investigation (paragraph 1) by establishing a number of criteria, including that of "the interests of justice." This criterion, conceived as a weighting criterion, allows the Prosecutor to refuse to open an investigation even if all the other criteria are present. It therefore constitutes the bastion of the discretionary power of the Prosecutor. In practice, no refusal to open an investigation has ever been based on the criterion of the "interests of justice." With the exception of the situations failing to meet the preliminary admissibility criteria, the refusals to open an investigation were based on the admissibility test and precisely on the gravity criterion. Does this mean that the Prosecutor himself or herself restricts his or her discretionary power by concentring on other criteria? This article seeks to demonstrate that the objectivation process to which the Prosecutor submitted himself or herself in taking into account these two criteria (interests of justice and gravity) has been damaging to the impartiality image that he or she wishes to project, necessitating adjustments. Therefore, the Prosecutor has minimized the use of the application of the criterion of interests of justice to the benefit of the gravity criterion, in favour of a strict interpretation of the "interests of justice" (I), while taking into account this criterion through diverted strategies, resulting in more difficult control and debates on his or her choices (II). We propose that considering the type of conflictuality the Prosecutor must face, it is necessary to question oneself on the

Candidate au doctorat à l'Université Laval. Ses recherches portent sur le critère de gravité et les politiques de poursuite du Procureur de la Cour pénale internationale. 
evolution of the spectrum of the interests of justice criterion, and thus of its taking into account in the choice to open an investigation (III).

Una de las grandes innovaciones en el dominio de la justicia internacional penal, a excepción del mismo acceso a un tribunal permanente, es la consagración, en su seno, de un Fiscal independiente. Esta independencia es objeto sin embargo de una regulación hecha particularmente a través del artículo 53 del Estatuto de Roma de la Corte penal internacional, titulado "Apertura de una investigación". En efecto, éste circunscribe la acción del Fiscal desde la fase de apertura de la investigación (párrafo 1) estableciendo un cierto número de criterios, del que están el del "Intereses de la justicia ". Este criterio, pensado como un criterio de ponderación, le permite al Fiscal negarse a abrir una investigación aunque el conjunto de otros criterios está presente. Constituye así el bastión del poder discrecional del Fiscal. En la práctica, ninguna negativa de apertura de investigación jamás se basó en el criterio de "Intereses de la justicia". Ahora bien, a excepción de las situaciones que no pasaban la prueba de admisibilidad, las negativas de apertura de investigación se basaron en la prueba de la admisibilidad y precisamente en el criterio de la gravedad. ¿Esto significa que el Fiscal mismo restringe su poder discrecional concentrándose sobre otros criterios? Nuestro esfuerzo pretende demostrar que el proceso de objetivación al cual se entregó el Fiscal en la toma de consideración de estos dos criterios (intereses de la justicia y la gravedad) fue perjudicial para la imagen de imparcialidad querida por este último, necesitando ajustes de su parte. Así, él minimizó la utilización de la aplicación del criterio de los intereses de la justicia en provecho del de la gravedad a favor de una interpretación estricta de "Intereses de la justicia" (I), tomando en consideración este criterio a través de estrategias apartadas que devuelven el control y los debates relativos a sus elecciones más complicadas (II). Ahora bien, pensamos que respecto al tipo de conflictividad al cual debe hacer frente, es necesario interrogarse sobre una evolución del espectro del criterio de los intereses de la justicia, y pues de su toma en cuenta en la elección de apertura de una investigación (III). 
«Ultimately a savvy international prosecutor is someone who, like all good politicians, realizes that not anything needs to be said but that the art of politics requires confrontation. "

Frédéric Mégret «Beyond "Gravity”: For a Politics of International Criminal Prosecutions » (2013) 107 Am Soc Intl L Proc 428 à la p 431 .

« Just as military solution to 'war' may not bring us peace, an exclusive focus on 'legal' needs and interests may not bring us justice. This is true, whether we like it or not. "

Carrie Menkel-Meadow, " Practicing 'in the Interests of Justice' in the Twenty-First Century: Pursuing Peace as Justice» (2001) 70 Fordham L Rev 1761 à la p 1174.

Une des grandes innovations dans le domaine de la justice internationale pénale, à l'exception de l'avènement même d'une cour permanente, est la consécration, en son sein, d'un Procureur indépendant. Cette création fut l'objet de nombreux débats lors des travaux préparatoires du Statut de Rome de la Cour pénale internationale $^{1}$. Ces discussions se sont reflétées dans les différentes évolutions du texte, à travers la problématique de l'étendue des pouvoirs accordés au Procureur par les États. Ainsi, au cœur du premier projet de statut de la Cour pénale internationale (ci-après CPI ou la Cour) préparé par la Commission du droit international (CDI), il n'existait que deux moyens de renvoi d'une situation devant la Cour : par les États ${ }^{2}$ ou par le Conseil de sécurité ${ }^{3}$. La possibilité d'un renvoi proprio motu d'une situation par le Procureur concentra les premières tensions entre les États ${ }^{4}$. Les partisans et les opposants d'un tel pouvoir utilisèrent la même trame argumentative ${ }^{5}$ pour débattre de ce texte, à savoir le risque de politisation de la $\operatorname{Cour}^{6}:$ soit par les renvois exclusifs d'entités politiques que sont les États et le Conseil de sécurité, soit par les influences subies par le Procureur dans le cadre d'un renvoi proprio motu. Finalement, inscrite au sein de l'article 15 du Statut de Rome, la possibilité de renvoi d'une situation par le Procureur sans contrôle d'un organe politique consacre une indépendance inédite pour un Procureur international ${ }^{7}$. Pour autant, la possibilité d'une telle autonomie dans ses

$1 \quad$ Statut de Rome de la Cour pénale internationale, 17 juillet 1998, 2187 RTNU 3 (entrée en vigueur : $1^{\mathrm{er}}$ juillet 2002) [Statut de Rome ou Statut].

Ce renvoi est consacré au sein de l'article 14 du Statut de Rome.

Ce renvoi est consacré à l'article 13 du Statut de Rome.

4 Allison M Danner, « Enhancing the Legitimacy and Accountability of Prosecutorial Discretion at the International Criminal Court» (2003) 97 Am J Intl L 510 à la p 513.

$5 \quad$ Ibid à la p 514.

6 Comme nous allons le voir tout au long de notre démonstration, cet argument de politisation, quel que soit les activités du Procureur, reste le plus fréquent.

7 Pour une analyse de l'indépendance des Procureurs au sein du Tribunal militaire international de Nuremberg et des Tribunaux pénaux internationaux, voir William Schabas, « Prosecutorial Discretion v Judicial Activism at the International Criminal Court » (2008) 6:4 J Intl Crim Justice 731 aux pp 73234 [Schabas, « Discretion »]. 
choix a été contrebalancée par des mécanismes de contrôle au sein du Statut. Ainsi, il est prévu un contrôle par une Chambre préliminaire dans l'article 15(3) et le contrôle politique n'est pas totalement absent du Statut puisque l'article 16 autorise le Conseil de sécurité à suspendre une enquête pour une période de douze mois renouvelable au moyen de l'adoption d'une résolution sur la base du Chapitre VII de la Charte des Nations Unies.

Le pouvoir discrétionnaire du Procureur fait également l'objet d'un autre encadrement: celui de l'article 53, intitulé «Ouverture d'une enquête ». En effet, celui-ci circonscrit l'action du Procureur dans le cadre de l'ouverture d'une enquête (paragraphe 1) et dans le cadre de poursuites (paragraphe 2), prévoyant également des procédures de contrôle (paragraphe 3 ) et la possibilité pour le Procureur de revenir sur sa décision (paragraphe 4). Avant d'examiner en détail cet article et sa portée au regard du pouvoir discrétionnaire du Procureur, il est nécessaire d'éclairer la relation entre l'article 15 et l'article 53. En effet, ces deux articles semblent évoluer de manière parallèle ${ }^{9}$ : un contrôle sous l'article 15 en cas de situation renvoyée proprio motu et un contrôle sous l'égide de l'article 53 pour un renvoi du Conseil de sécurité ou par un État. Qu'en est-il ? Sans exposer avec détail les arguments des débats doctrinaux en l'espèce, nous nous fonderons sur les interprétations convergentes faites par Fernandez et Pacreau ${ }^{10}$ et Turone ${ }^{11}$, qui explicitent que l'article 53 est applicable à toutes les situations, quel que soit le mécanisme de renvoi, s'appuyant ainsi sur la décision de la Chambre préliminaire II concernant la situation au Kenya ${ }^{12}$.

Ainsi, l'article 53, premier du Chapitre V intitulé «Enquêtes et poursuites » qui consacre les pouvoirs exclusifs d'ouverture d'enquête confiés au Procureur ${ }^{13}$, constitue un encadrement de ce pouvoir. Avant toute autre considération, il est nécessaire de rappeler que la sélection dans l'ouverture des enquêtes constitue une «nécessité fonctionnelle ${ }^{14}$ pour la Cour et le Procureur qui ont compétence sur potentiellement un nombre infini de situations au sein desquelles les crimes poursuivis par la Cour ont eu lieu (même en tenant compte des limites ratione temporis $^{15}$, materiae $^{16}$ et personae ${ }^{17}$ ). Toute cette évaluation se fait dans le cadre d'un

\footnotetext{
$8 \quad$ Charte des Nations unies, 26 juin 1945, RT Can 1945 nº 7.

9 Julian Fernandez et Xavier Pacreau, dir, Statut de Rome de la Cour pénale internationale : commentaire article par article, Paris, Pedone, 2012 à la p 1177 [Fernandez et Pacreau]. En l'espèce, il est expliqué que la prolongation des négociations sur l'article 15 (adopté dans les dernières heures des discussions) a eu pour conséquence le peu d'attention accordée, par manque de temps, à l'harmonisation des deux articles.

$10 \quad$ Ibid à la p 1180 .

11 Giuliano Turone, «Powers and Duties of the Prosecutor » dans Antonio Cassese, Paola Gaeta et John R W D Jones, dir, The Rome Statute of the International Criminal Court: A Commentary, New York, Oxford University Press, 2002 à la p 1147 [Turone].

12 Fernandez et Pacreau, supra note 9 à la $\mathrm{p} 1181$.

13 Ibid à la p 1178.

14 Alana Tiemessen, « The International Criminal Court and the Politics of Prosecutions » (2014) 18:3 Intl JHR 444 à la p 448.

15 Statut de Rome, supra note 1, art 11.

16 Ibid, art 5.
} 
examen préliminaire, défini par l'article 53(1) à travers les dispositions des alinéas a) à c).

Néanmoins, ce processus constitue aussi un premier contrôle du pouvoir discrétionnaire du Procureur. L'identification d'une situation est l'étape préliminaire à l'identification des affaires, ainsi une situation "refers to larger areas of conflict, in which the OTP [Office of the Prosecutor ou Bureau du procureur, NDLR] investigates and builds up several case hypotheses ${ }^{18} \gg$. Afin d'ouvrir une enquête qui permettra la poursuite d'affaires, le Procureur doit appliquer un certain nombre de critères aux situations renvoyées durant l'examen préliminaire ${ }^{19}$. En premier lieu, le Procureur doit examiner si les informations qui lui sont fournies constituent «une base raisonnable pour croire qu'un crime relevant de la compétence de la Cour a été ou est en voie d'être commis ${ }^{20} »$, ce qui correspond à un test d'admissibilité dont le degré de preuve est faible ${ }^{21}$. Ce premier test constitue un «assessment based on an objective and specific indicia-on whether there is a realistic prospect that an investigation would lead to a prima facie case about a given crime and its perpetrators ${ }^{22} \gg$. Cette évaluation ne fait pas appel au pouvoir discrétionnaire, mais se base sur des considérations objectives. Le deuxième volet du test, qu'est la recevabilité au regard de l'article $17^{23}$, comporte deux considérations. Celui-ci demande de vérifier la complémentarité (l'absence de poursuites ou de volonté de poursuites par l'État) en se basant sur les dispositions de l'article 17(1)(a) à 17(1)(c)) et la gravité de la situation. Ce dernier critère n'est pas défini dans le Statut. Une fois ces deux premiers volets passés avec succès, l'article 53 prévoit que le Procureur peut néanmoins refuser d'ouvrir une enquête " [s]'il y a des raisons sérieuses de penser, compte tenu de la gravité du crime et des intérêts des victimes, qu'une enquête ne servirait pas les intérêts de la justice ${ }^{24} »$, disposition qualifiée par Schabas de «vague expression that

17 Elle concerne les crimes commis par les ressortissants d'un État partie (article 12(2)(b)), les personnes ayant commis des crimes sur le territoire d'un État partie (article 12(2)(a)) ou dans le cadre d'une situation déférée par le Conseil de sécurité (article 13) ou d'un État non-partie mais qui donne une compétence à la Cour (article 12(3)). Statut de Rome, supra note 1, art 12.

18 Kai Ambos et Ignaz Stegmiller, « Prosecuting International Crimes at the International Criminal Court: Is There a Coherent and Comprehensive Prosecution Strategy? » (2013) 59:4 Crim L \& Soc Change 415 à la p 419 .

19 Pour une description des étapes de l'examen préliminaire, voir la page du site de la Cour pénale internationale, en ligne : CPI

$<$ https://www.icc-

cpi.int/fr_menus/icc/structure $\% 20$ of $\% 20$ the $\% 20$ court/office $\% 20$ of $\% 20$ the $\% 20$ prosecutor/comm $\% 20$ an $\mathrm{d} \% 20 \mathrm{ref} /$ Pages/communications $\% 20$ and $\% 20$ referrals.aspx $>$.

20 Statut de Rome, supra note 1, art 53(1)(a).

21 Fernandez et Pacreau, supra note 9 à la p 1191.

22 Turone, supra note 11 à la p 1152.

23 Unanimement, la doctrine s'est questionnée sur l'utilisation du terme « affaire » dans cette partie de l'article, pour conclure qu'il est « desirable to construe the term as meaning a situation ». William Schabas, The International Criminal Court: A Commentary on the Rome Statute, New York, Oxford University Press, 2010 à la p 660 [Schabas, International Criminal Court].

24 Statut de Rome, supra note 1, art 53(1)(c). 
effectively leaves him with a free hand ${ }^{25}$ ". Il est nécessaire de comprendre l'étendue du pouvoir discrétionnaire donné par cet alinéa : même en cas de renvoi par le Conseil de sécurité ou par un État, le Procureur peut refuser d'ouvrir une enquête, à l'aide du critère de pondération que sont "les intérêts de la justice", consacrant ainsi véritablement son indépendance. Proposition britannique fondée sur l'existence d'une telle considération dans les systèmes nationaux de common law ${ }^{26}$, l'expression «les intérêts de la justice » n'a paradoxalement pas été définie dans le Statut, du fait de l'absence de consensus des participants sur les circonstances qui pourraient amener le Procureur à refuser d'ouvrir une enquête ${ }^{27}$. Les «intérêts de la justice» sont mentionnés deux fois dans l'article 53 (au stade de l'ouverture de l'enquête et des poursuites). L'analyse en l'espèce ne portera que sur ceux mentionnés à l'article 53(1)(c) relatif à l'étape de l'ouverture de l'enquête.

Ce sont les deuxième et troisième étapes qui concentrent l'exercice du pouvoir discrétionnaire du Procureur au regard de critères non définis qui laissent place à l'exercice de son jugement. Il est intéressant de tenter de percevoir comment il utilise cette indépendance à travers l'application des critères, car cette utilisation est aussi marquée par un enjeu plus grand qu'est la légitimité ${ }^{28}$ de cet organe de la Cour sur la scène internationale, indispensable à la crédibilité de l'institution. Ces considérations sont d'ailleurs prises en compte dans le Document de politique générale relatif aux examens préliminaires, publié par le Bureau du Procureur (ciaprès le Bureau) en $2013^{29}$, qui indique que l'application des critères relatifs à l'examen doit se faire selon trois principes: l'indépendance, l'impartialité et l'objectivité ${ }^{30}$.

Le critère le plus susceptible de porter préjudice aux principes guidant l'action du Procureur est celui des intérêts de la justice, du fait de son absence de définition et des divergences de points de vue qu'il a suscitées lors des travaux préparatoires, ainsi que de son rôle ultime dans l'examen préliminaire. Comme nous l'avons déjà mentionné, un autre critère retient l'attention à la lecture de l'article 53(1) : celui de la gravité. En effet, celui-ci est présent deux fois : dans le

25 William Schabas, "Victor's Justice: Selecting "Situations" at the International Criminal Court » (2010) 43 J Marshall L Rev 535 à la p 541 [Schabas, « Victor's Justice »].

26 Schabas, International Criminal Court, supra note 23 à la p 663.

27 Ibid.

28 La légitimité est définie comme «qualité d'une institution [...] qu'elle tire de sa conformité à des valeurs jugées essentielles par le milieu dans lequel elle se situe ». En ce sens, les qualités nécessaires à la légitimité dont nous parlons ici sont celles évoquées dans la phrase suivante à savoir l'indépendance, l'impartialité et l'objectivité. Jean Salmon, Dictionnaire de droit international public, Bruxelles, Bruylant, 2001 à la p 644.

29 Bureau du Procureur, Document de politique générale relatif aux examens préliminaires (novembre 2013) à la p 7 en ligne : CPI <Bureau du Procureur, Document de politique générale relatif aux examens préliminaires, La Haye (novembre 2013), en ligne : CPI < https://www.icc-cpi.int/en_menus/ icc/structure $\% 20$ of $\% 20$ the $\% 20$ court/office $\% 20$ of $\% 20$ the $\% 20$ prosecutor/policies $\% 20$ and $\% 20$ strategies /Documents/OTP_Policy_Paper_on_Preliminary_Examinations_November_2013_FRA.PDF> [Bureau du Procureur, Document de politique générale relatif aux examens préliminaires].

30 Les deux premiers principes sont consacrés à l'article 42 du Statut de Rome. On peut considérer l'objectivité comme un moyen de parvenir à la réalisation des deux principes évoqués précédemment. 
cadre du test d'admissibilité de l'article 17 de l'alinéa b), paragraphe 1 de l'article 53 et dans le cadre de la prise en compte des «intérêts de la justice » à l'alinéa c). Sa mention dans le cadre de l'article 17 est restée pratiquement la même entre le projet de la CDI de 1994 et son adoption dans le Statut ${ }^{31}$, démontrant l'accord des États sur la nécessité de porter des affaires d'une " gravité suffisante ${ }^{32}$ » et a été rajouté dans la formulation de l'article 53(1)(c) qui, au départ, ne précisait que la prise en compte des intérêts des victimes dans la considération des intérêts de la justice ${ }^{33}$. On peut supposer de ces deux remarques que même si la gravité n'a pas été définie, son inclusion s'est avérée assez rassurante pour les États dans le contrepouvoir que ce critère pouvait exercer face à l'énorme aspect discrétionnaire laissé par l'expression « intérêts de la justice».

Dans la pratique, cette vision semble avoir porté ses fruits. En effet, aucun refus d'ouverture d'enquête par le Procureur ne s'est jamais fondé sur le critère des « intérêts de la justice». Or, à l'exception des situations ne passant pas le test d'admissibilité, les refus d'ouverture d'enquête se sont fondés sur le test de la recevabilité et précisément sur le critère de la gravité ${ }^{34}$. Peut-on donc qualifier d'objective la décision d'ouvrir une enquête? Plus précisément, comment ces deux critères sont-ils utilisés par le Procureur dans le cadre de l'ouverture des enquêtes, sous l'égide de ses principes d'impartialité, d'indépendance et d'objectivité ? Cette problématique nous poussera à comprendre comment le critère des "intérêts de la justice », prévu à l'article 53 du Statut de Rome, guide le Procureur de la CPI dans le processus de décision quant à l'ouverture d'une enquête, et comment ce critère s'articule avec celui de la "gravité ».

Notre démarche vise à démontrer que le processus d'objectivation auquel s'est livré le Procureur dans la prise en compte de ces deux critères a été nuisible à l'image d'impartialité voulue par ce dernier, nécessitant des ajustements de sa part. Ainsi, il a minimisé l'utilisation de l'application du critère des intérêts de la justice au profit de celui de la gravité à la faveur d'une interprétation stricte des " intérêts de la justice » (I), tout en prenant en compte ce critère par l'intermédiaire de stratégies détournées rendant le contrôle et les débats relatifs à ses choix plus compliqués (II). Or, nous pensons qu'au regard du type de conflictualité auquel il doit faire face, il est nécessaire de se questionner sur une évolution du spectre du critère des intérêts de la justice, et donc de sa prise en compte dans le choix d'ouverture d'une enquête (III).

\footnotetext{
Otto Triffterer et Kai Ambos, dir, Rome Statute of the International Criminal Court: A Commentary,



32 Statut de Rome, supra note 1, art 17(1)(d).

33 Turone, supra note 11 à la p 1153.

34 Ces cas sont ceux de la situation en Iraq et des Comores, dont les détails des évaluations peuvent être trouvés sur le site de la CPI, en ligne : <https://www.icc-cpi.int/about/otp?ln=fr>.
} 


\section{L'affirmation d'une interprétation stricte du critère des intérêts de la justice : une lecture de l'article 53 à la lumière des principes d'objectivité et d'impartialité}

Ce qui sous-tend l'interprétation par le Procureur et par une partie de la doctrine est la volonté d'objectivation du pouvoir discrétionnaire du Procureur afin de contrer tout argumentaire de partialité. Ainsi, une lecture restreinte du critère des intérêts de la justice a été développée par le Procureur et une partie des acteurssatellites de la justice internationale (A), alors que le critère de gravité a vu son spectre définitionnel évoluer au gré de la politique du Bureau relative aux examens préliminaires et de la jurisprudence, laissant une porte à l'exercice de son pouvoir discrétionnaire (B).

A. L'affirmation d'une lecture restreinte des «intérêts de la justice»: l'avènement d'une justice rétributive

L'interprétation à donner au critère des « intérêts de la justice » est devenue une prise de position sur le concept de justice à faire prévaloir. L'argumentaire s'est développé à travers les documents constitutifs de la politique du Bureau (1) et a trouvé écho chez une partie de la doctrine et des organisations non gouvernementales (2).

1. UNE VISION ÉLABORÉE DANS LES DOCUMENTS DE POLITIQUE GÉNÉRALE DU BUREAU DU PROCUREUR

L'analyse de l'interprétation des intérêts de la justice se fera en examinant le Document de politique relatif aux intérêts de la justice ${ }^{35}$, dont les termes sont repris dans le Document de politique générale relative aux examens préliminaires, publié en $2013^{36}$. Il est nécessaire de rappeler que les «intérêts de la justice » constituent un critère de pondération ${ }^{37}$ au terme de l'examen préliminaire; il n'est pris en considération que si les tests de recevabilité et d'admissibilité sont positifs.

En septembre 2007, le Bureau du Procureur publie un document intitulé Policy Paper on the Interests of Justice, ayant pour but d'éclairer son approche concernant ce critère. L'espoir d'une telle lumière s'éteint dès l'introduction. En effet, il est notifié qu'il ne sera pas discuté en détail des facteurs et circonstances permettant

35 Bureau du Procureur, Document de politique relatif aux intérêts de la justice (septembre 2007), en ligne : CPI

<https://www.icc-cpi.int/nr/rdonlyres/772c95c9-f54d-4321-bf09-73422bb23528/143640/

iccotpinterestsofjustice.pdf $>$ [Bureau du Procureur, Policy Paper on the Interests of Justice].

36 Bureau du Procureur, Document de politique générale relatif aux examens préliminaires, supra note 29.

37 Ibid à la p 3. 
l'élaboration d'une grille de lecture favorable à l'analyse du critère ${ }^{38}$. Néanmoins, trois principes doivent guider l'application de ce critère par le Procureur. En premier lieu, il existe une présomption favorable aux enquêtes et aux poursuites rendant l'application de l'article 53(1)(c) exceptionnelle. De plus, le pouvoir discrétionnaire du Procureur s'exerce à la lumière des buts du Statut en référence à l'article 31 de la Convention de Vienne sur le droit des traités ${ }^{39}$, rappelant ainsi le rôle de la CPI dans la lutte contre l'impunité, celui-ci constituant conséquemment la ligne directrice du Bureau. Enfin - et c'est le plus intéressant à nos yeux -, il est clairement indiqué que les considérations de paix ne font pas partie du mandat du Procureur. Le Procureur vient en l'espèce clairement affirmer sa position dans le débat paix/justice : les ressorts politiques relatifs aux considérations de paix sont sous la coupe d'autres institutions et n'interfèrent pas dans son analyse des intérêts de la justice.

Il est nécessaire ici de s'interroger sur le besoin d'une affirmation aussi tranchée au sein du Bureau. Notre position en l'espèce est de considérer que cette posture a été envisagée comme nécessaire à la construction de la légitimité du travail du Bureau, en s'inscrivant dans un point de vue purement légaliste. Ainsi, selon ce point de vue, le droit et la politique internationale doivent être séparés afin d'éviter d'alimenter toute accusation de politisation des travaux du Bureau. On peut noter en ce sens la volonté de rassurer voulue au sein du document («[t]he Office of the Prosecutor understands the interest raised by Article 53(1) (c) and 53(2) (c) $\left.{ }^{40} »\right)$ et l'emphase mise sur le rôle de contrôle de la Chambre préliminaire comme organe de révision de la décision ${ }^{41}$.

La latitude laissée par un tel critère au pouvoir discrétionnaire du Procureur est telle que ce dernier fait lui-même la promotion d'une utilisation restrictive, en s'appuyant à la fois sur une conception positiviste du droit, sur la prise en compte des préoccupations externes (dont les positons des organisations non gouvernementales (ONG) considérées dans le développement suivant) et sur les garde-fous institutionnels, que sont les procédures et le rôle de la Chambre préliminaire, prévus à l'article 53(3) et dont la portée sera discutée dans la deuxième partie de notre démonstration. Par ailleurs, en décrivant les différents paramètres à prendre en compte dans l'évaluation des intérêts de la justice sous l'article 53(1)(c) (il ne retient que gravité et intérêts des victimes tels que mentionnés dans la lettre du texte), le Procureur vient rappeler que le critère de gravité est premier dans le test d'admissibilité et qu'il est central dans sa décision d'enquêter en imposant une présomption favorable aux poursuites (nous reviendrons plus explicitement sur la structure de réflexion entourant cette affirmation dans la partie IB), rendant exceptionnelle la possibilité d'une affirmation selon laquelle l'ouverture d'une enquête serait contraire aux intérêts de la justice.

\footnotetext{
38 Bureau du Procureur, Document de politique relatif aux intérêts de la justice, supra note 35 à la p 1.

39 Convention de Vienne sur le droit des traités, 23 mai 1969, 1155 RTNU 331 (entrée en vigueur : 27 janvier 1980) [Convention de Vienne].

40 Bureau du Procureur, Document de politique relatif aux intérêts de la justice, supra note 35 à la p 9.

41 Le rôle de la Chambre est mentionné trois fois durant les neuf pages du document.
} 
Dans son Document de politique relatif aux intérêts de la justice de 2007, le Procureur en vient par ailleurs à se demander s'il existe d'autres critères à prendre en compte dans les intérêts de la justice que la gravité et les intérêts des victimes. Il évoque ainsi les autres processus de justice alternative ${ }^{42}$ et les processus de paix ${ }^{43}$. La politique portée par le Bureau en la matière affirme le rôle central des poursuites pénales dans le cadre de la justice transitionnelle, les autres mécanismes, dont les Commissions vérité et réconciliation (CVR) sont considérés comme complémentaires à la primauté des poursuites pénale ${ }^{44} \mathrm{~S}$. Il renvoie par ailleurs les considérations de paix au Conseil de sécurité, invoquant l'article 16 du Statut, qui permet à ce dernier de suspendre une enquête. Le Procureur précise que le critère "should not be conceived of so broadly as to embrace all issues related to peace and security ${ }^{45} "$. Cette interprétation s'est effectivement concrétisée dans la pratique.

À la lumière des développements précédents, nous pouvons dire que la justice dont les intérêts doivent être préservés par l'ouverture d'une enquête est une justice de nature rétributive, le Procureur percevant les autres aspects de la justice transitionnelle comme des compléments et non comme des mesures toutes aussi importantes qui pourraient être mises en danger par une enquête. De même, les considérations de paix sont exclues et laissées aux mains du Conseil de sécurité, dans un esprit de « division du travail » que nous étaierons plus tard.

Les tenants et aboutissants de cette vision restrictive ont été développés par les ONG et la doctrine.

\section{UNE INTERPRÉTATION LÉGALISTE DU TERME «JUSTICE » DÉVELOPPÉE ET DÉFENDUE PAR UNE PARTIE DES COMMENTATEURS DE LA JUSTICE INTERNATIONALE PÉNALE}

La vision restrictive de la justice portée par le Procureur a été explicitée par un certain nombre d'acteurs satellites de la justice internationale pénale : les ONG et une partie de la doctrine. L'ensemble de ces acteurs a expliqué pourquoi il ne fallait pas tenir compte de tous les mécanismes de justice transitionnelle dans la conception de la justice et pourquoi le Procureur devait se détacher des enjeux nationaux quand il devait choisir d'ouvrir une enquête.

Ainsi, en 2004, soit trois ans avant le document de politique du Procureur relatif aux «intérêts de la justice», le Procureur a consulté les ONG. On notera que les étapes de l'argumentaire, développées notamment par Human Rights Watch de manière très précise ${ }^{46}$, ont été reprises de façon plus concise au sein du document

\footnotetext{
Bureau du Procureur, Document de politique relatif aux intérêts de la justice, supra note 35 à la p 7. Ibid à la $\mathrm{p} 8$.

44 Ibid.

45 Ibid.

46 Human Rights Watch, The Meaning of " the Interests of Justice " in Article 53 of the Rome Statute (juin 2005), en ligne : HRW <https://www.hrw.org/news/2005/06/01/meaning-interests-justice-article53-rome-statute $>[\mathrm{HRW}]$.
} 
présenté en 2007. Premièrement, les objectifs du préambule du Statut définissent la mission de la Cour (la lutte contre l'impunité, la prévention des crimes, la compétence de la Cour pour les crimes les plus graves, la complémentarité) et font du rôle du Procureur en matière de poursuites une des clés de voûte de la Cour ${ }^{47}$. Par ailleurs, selon la Fédération internationale des ligues des droits de l'homme ${ }^{48}$, les intérêts de la justice doivent être compris comme incluant les intérêts de l'institution judiciaire (bonne administration), les droits de la défense et les composantes d'un procès équitable $^{49}$. Ce n'est que si une enquête porte atteinte à ces composantes qu'elle doit être considérée comme contraire aux intérêts de la justice.

Les deux ONG rappellent également les dispositions du droit international excluant les amnisties pour les crimes les plus graves ${ }^{50}$. La prise en compte des enjeux de paix et de sécurité est également à exclure car un risque trop important de pression politique sur le Procureur pourrait en résulter, de deux manière « (a) risk being mired in making political judgments that would ultimately undermine his work; (b) risk subjecting the OTP to enormous political pressures and attempted manipulation by governments and rebel groups ${ }^{51} »$.

Dans une perspective cohérente, la notion d' « intérêts des victimes » qui doit être prise en compte dans la balance des intérêts de la justice est évaluée de manière restrictive. Ainsi,

it is not a justice interest whether some victims believe a prosecution will hurt chances for a peace settlement: their needs for justice, whether great or small, will remain unchanged. While the Prosecutor should always be receptive to hearing the views of victims, those views are not all necessarily relevant to a determination under Article $53^{52}$.

Une partie de la doctrine a également soutenu cette interprétation restrictive. Comme nous l'avons vu précédemment, les débats doctrinaux relatifs aux intérêts de la justice se sont en fait transformés en position sur les mesures de justice les plus appropriées à la sortie d'un conflit. Ainsi, les bienfaits de la justice pénale sont rappelés par de nombreux auteurs ${ }^{53}$. Les potentielles finalités d'une telle justice sont

$47 \quad$ Ibid à la p 5.

48 Fédération internationale des ligues des droits de l'homme, Réflexions sur la notion « intérêts de la justice " au terme de l'article 53 du Statut de Rome (2005) à la p 3, en ligne: FIDH $<$ https://www.fidh.org/IMG/pdf/cpi20062005f.pdf $>$ [FIDH].

49 Ibid à la $\mathrm{p} 3$.

50 HRW, supra note 46 à la p 8; FIDH, supra note 48 à la p 8.

51 HRW, supra note 46 à la $\mathrm{p} 9$.

52 Ibid à la $\mathrm{p} 13$.

53 Thomas H Clark, « The Prosecutor of the International Criminal Court, Amnesties and the "Interests of Justice": Striking a Delicate Balance » (2005) 4:2 Washington University Global Studies Law Review 389 [Clark]; Kate Allan, « Prosecution and Peace: A Role for Amnesty Before the ICC? » (2011) 39:1 Denv J Intl L \& Pol'y 239 [Allan]; Diego A Arcarazo, Russell Buchan et Rene Ureña, « Beyond Justice, Beyond Peace? Colombia, the Interests of Justice, and the Limits of International Criminal Law » (2015) 26:2 Crim LF 291 [Arcarazo, Buchan et Ureña]; Drazan Đukic, « Transitional Justice and the International Criminal Court-In "the Interests of Justice”? » (2007) 89 RICR 691 [Đukic]. 
mises en exergue : mettre fin aux cycles de violence, participer à renforcer l'état de droit, avoir un effet dissuasif, participer à la réconciliation ou encore individualiser les responsabilités ${ }^{54}$. Concernant l'interprétation du critère des intérêts de la justice, pour Dukic, les «intérêts de la justice » devant être pris en compte dans l'article 53(1)(c) sont ceux relatifs à une justice perçue de manière rétributive puisque les seuls critères envisagés sont ceux de la gravité et des intérêts des victimes ${ }^{55}$. Il différencie cette approche d'une qui serait plus large dans l'article 53(2)(c) relatif aux poursuites et qui est formulée ainsi : «[p]arce que poursuivre ne servirait pas les intérêts de la justice, compte tenu de toutes les circonstances, y compris [...]» [nos soulignements]. Une interprétation large de la justice serait donc réservée au moment décisionnel lié aux poursuites. Ainsi le stade de la décision de l'ouverture de l'enquête semble être trop précoce pour laisser un poids trop important au critère des intérêts de la justice.

Ce rappel des intérêts de la justice rétributive s'est également manifesté par une mise en lumière prospective des dilemmes auxquels le Procureur serait confronté s'il accepte la prise en compte de mécanismes alternatifs de justice dans les intérêts de la justice et en particulier les amnisties. Ainsi, Allan craint que de la prise en compte des amnisties résulte la «cristallisation» d'une norme autorisant les amnisties ${ }^{56}$. Elle explicite trois problématiques issues d'une telle pratique : premièrement, comment le Procureur peut-il décider si le modèle de justice choisi par l'État est approprié ou non, et donc si des poursuites sont nécessaires? Deuxièmement, si le Procureur embrasse ce rôle, il s'ingère dans les choix politiques nationaux et enfin, il pourrait faire émerger des normes en matière d'amnisties risquant d'entrer en conflit avec certaines obligations de poursuites inscrites en droit international ${ }^{57}$. L'argument final invoqué est celui d'une analyse de l'article 16 autorisant le Conseil de sécurité à suspendre une enquête comme étant la marque d'une volonté de «division des tâches» du Statut ${ }^{58}$. Ainsi selon cette vision, la sphère internationale bénéficie d'une organisation comparable au Taylorisme : la justice pour la CPI et la politique pour le Conseil de sécurité, qui reprend la main quand le ratio politique/justice augmente.

Pour conclure, nous ajouterons que le choix d'une interprétation restrictive correspond à une justification de l'existence même de la fonction et de l'utilité d'une cour internationale pénale, ainsi qu'une volonté de promotion de son impartialité. Cette minimisation de l'utilisation des intérêts de la justice comme filtre des enquêtes a des conséquences sur un autre critère, qui lui, devient prépondérant dans la justification de l'action du Procureur : celui de la gravité.

54 Clark, supra note 53 aux pp 402- 403.

Đukic, supra note 53.

Allan, supra note 53 à la p 292.

Ibid aux pp 292- 293.

58 Arcarazo, Buchan et Ureña, supra note 53 p 314. 


\section{B. La gravité : un critère à l'objectivité... élastique ?}

La place du critère de gravité est détaillée dans le document de politique relatif aux intérêts de la justice, puisqu'avec les intérêts des victimes, ce dernier est clairement spécifié comme un élément central dans la démarche de sélection quant au refus d'ouverture d'une enquête ${ }^{59}$. Il est toutefois nécessaire de noter la tautologie utilisée par le Procureur dans son analyse de la prise en compte de ce critère : le Statut a été créé pour poursuivre les crimes les plus graves et afin de valider le critère d'admissibilité, ces crimes doivent être «suffisamment graves ${ }^{60}$ ». Ainsi, ce critère d'admissibilité est déjà évalué comme positif quand les intérêts de la justice doivent être analysés donc cela signifie que la situation comporte des crimes suffisamment graves pour qu'il faille les poursuivre. Ce raisonnement circulaire empêche tout questionnement sur les composantes du critère de gravité en tant que telles et donc sur leur mise en balance dans les intérêts de la justice. Dans le critère de gravité doivent être pris en compte: l'échelle des crimes, leur nature, le mode opératoire et leur impact $^{61}$, comme le spécifie le Document de politique générale relatif aux examens préliminaires de $2013^{62}$.

XX. La centralité du critère tel qu'exposée et utilisée par le Procureur en faitelle un critère objectif qui participerait à l'impartialité du Procureur ? Comme le rappellent différents auteurs ${ }^{63}$, ce critère a été perçu comme non controversé dans les travaux préparatoires du Statut de Rome et plutôt rassurant comme levier d'encadrement du pouvoir de sélectivité du Procureur. Cette vision pourrait expliquer sa double apparition au sein de l'article 53(1) dans les alinéas b) et c). En effet, le critère de gravité est utilisé comme un goulot d'étranglement nécessaire à l'acceptation d'un nombre de situations gérables par le bureau du Procureur ${ }^{64}$.

Non défini dans le Statut, ce critère n'a commencé à être utilisé qu'à partir de 2005 par le Procureur pour justifier ses choix d'ouverture d'enquêtes ${ }^{65}$. Il a notamment été invoqué pour expliquer le refus d'ouverture d'une enquête sur les allégations de crimes de guerre (traitements inhumains et meurtres) ${ }^{66}$ par des soldats britanniques en Iraq, lors du premier examen préliminaire dont cette situation a fait

59 Bureau du Procureur, Document de politique relatif aux intérêts de la justice, supra note 35 à la p 5.

60 Statut de Rome, supra note 1, art 17(1)(d).

61 Cour pénale internationale, Règlement du Bureau du Procureur, ICC-BD/05-01-09 (avril 2009), règle 29.

62 Bureau du Procureur, Document de politique générale relatif aux examens préliminaires, supra note 29 à la $\mathrm{p} 16$.

63 Mohamed M El Zeidy, «The Gravity Threshold Under the Statute of the International Criminal Court » (2008) 19:1 Crim LF 35 à la p 35 [El Zeidy]; Frédéric Mégret "Beyond "Gravity": For a Politics of International Criminal Prosecutions » (2013) 107 Am Soc Intl L Proc 428 à la p 428 [Mégret]; Schabas, « Discretion », supra note 7 à la $\mathrm{p} 7$.

64 Ibid à la $\mathrm{p} 37$.

65 Schabas, « Discretion », supra note 7 à la p 737.

66 Bureau du Procureur, Réponse du BDP concernant les communications reçues à propos de l'Irak (février 2006) à la p 8 , en ligne : CPI $<$ https://www.icc-cpi.int $>$ [Bureau du procureur, Réponse $d u$ BDP concernant les communications reçues à propos de l'Irak]. 
l'objet ${ }^{67}$, ainsi que la situation relative aux navires battant pavillon comorien, grec et cambodgien (dite "Affaire de la flottille de Gaza») ${ }^{68}$. Ainsi, dans la première situation, il est signifié par le Procureur Moreno-Ocampo que même si les crimes allégués tombaient bien sous la compétence de la Cour, ces derniers n'étaient pas assez graves au regard de trois autres situations dont la Cour était en charge à l'époque à savoir le Soudan, l'Ouganda et la République démocratique du Congo (RDC). Pour justifier cette décision, il compara le nombre de victimes potentielles sous sa juridiction en Iraq et celles des trois autres situations ${ }^{69}$, ce qui fit dire à certains observateurs que la démarche intellectuelle en l'espèce était faussé, car il « comparait des pommes et des oranges ${ }^{70}$ ».

Le critère a fait l'objet d'une interprétation pour la première fois par la Chambre préliminaire I dans l'affaire Lubanga Dyilo, dans la décision relative à la Requête du Procureur aux fins de la délivrance d'un mandat d'arrêt en vertu de l'article $58^{71}$ et indiquait que le critère de gravité de l'article 17 était atteint si trois considérations étaient prises en compte; à savoir en premier lieu, si le comportement mis en cause dans l'affaire est à grande échelle ou systématique et a provoqué l'indignation de la communauté internationale, si en deuxième lieu, les personnes soupçonnées entrent dans la catégorie des plus hauts dirigeants et enfin si la personne portant la plus haute responsabilité l'est du fait du rôle joué par son organisation dans la perpétration des crimes ou du fait de son rôle dans cette organisation quand les crimes sont commis ${ }^{72}$. Cette décision n'a pas été confirmée en appel et la Chambre a notamment rejeté le recours à l'indignation internationale comme critère à prendre en compte $^{73}$, sans pour autant étayer son interprétation du critère de gravité.

67 La situation en Iraq est actuellement soumise à un examen préliminaire suite à la décision de la Procureure Fatou Bensouda le 13 mai 2014. Voir «Le Procureur de la Cour pénale internationale, Fatou Bensouda, procède à un nouvel examen préliminaire de la situation en Irak », en ligne : CPI $<$ https:/www.icc-cpi.int/FR_Menus/icc/press\%20and\%20media/press\%20releases/pages/otpstatement-iraq-13-05-2014.aspx $>$ [Communiqué 2014].

68 Bureau du Procureur, Referral of the "Union of the Comoros " Under Articles 14 and 12(2)(a) of the Rome Statute arising from the 31 May 2010, Gaza Freedom Flotilla situation (14 mai 2013), en ligne : CPI <www.icc-cpi.int/iccdocs/otp/Referral-from-Comoros.pdf $>$ [Bureau du Procureur, Gaza Freedom Flotilla Situation].

69 Bureau du procureur, Réponse du BDP concernant les communications reçues à propos de l'Irak, supra note 66 aux pp 8-9.

70 Schabas, «Discretion », supra note 7 à la p 741.

71 Le Procureur c Thomas Lubanga Dyilo, ICC-01/04-01/06-8-US, Décision relative à la Requête du Procureur aux fins de délivrance d'un mandat d'arrêt en vertu de l'article 58 (24 février 2006) (Cour pénale internationale, Chambre préliminaire I), en ligne : CPI <https:/www.icc-cpi.int $>$ [Lubanga Dyilo].

72 Ibid aux pp 29-31.

73 Le Procureur c Thomas Lubanga Dyilo, ICC-01/04-169-tFRA, Arrêt relatif à l'appel interjeté par le Procureur contre la décision de la Chambre préliminaire I intitulée « Décision relative à la requête du Procureur aux fins de délivrance de mandats d'arrêt en vertu de l'article 58 » (13 juillet 2006) à la p 25 (Cour pénale internationale, Chambre d'appel), en ligne: CPI <https://www.icccpi.int/CourtRecords/CR2007_01744.PDF>. 
Après ces quelques détours jurisprudentiels, le critère a fait l'objet d'orientations par les Chambres préliminaires, dans la situation au Darfour ${ }^{74}$ et dans la situation au Kenya ${ }^{75}$. Ainsi, la Chambre préliminaire II explique concernant la situation au Kenya que l' " examen de la gravité des crimes peut être mené selon une approche tant quantitative et qualitative $\rangle^{76}$. La dimension qualitative est celle qui s'intéresse aux circonstances des crimes ${ }^{77}$. Les observateurs ont noté la potentielle élasticité de la prise en compte d'une approche qualitative de la gravité ${ }^{78}$. Par ailleurs, comme nous allons le voir dans le développement suivant, avec la situation des Comores, la gravité reste au cœur des considérations des Chambres préliminaires.

Pour Schabas :

[t]he "gravity" language strikes the observer as little more than obfuscation, a contrived attempt to make the determinations look objective and judicial. [...] they have found a legalistic formula enabling them to do the impossible, namely, to make what is inexorably a political decision but without making it look political ${ }^{79}$.

Mégret met en perspective ce constat en analysant que la difficulté d'explorer tous les enjeux de la gravité provient de l'incapacité des juristes à conceptualiser cette notion et notamment à explorer ses degrés, du fait du paradoxe inhérent à la justice internationale pénale :

it is, on the one hand, based on the idea that there is a degree of absolute consensus about certain values and the need to protect them, but on the other hand, this consensus seems to be based on a rather superficial agreement and tends to break down when confronted with the world's reality ${ }^{80}$.

Pour conclure, cette première partie peut offrir l'impression de l'inexistence de la prise en compte des « intérêts de la justice». Or, il est intéressant de voir que le Procureur a développé d'autres stratégies permettant de les prendre en compte, sans avoir à justifier ses choix.

74 Le Procureur c Bahar Idriss Abu Garda, ICC-02/05-02/09-243, Décision relative à la confirmation des charges (8 février 2010) (Cour pénale internationale, Chambre préliminaire I), en ligne: CPI $<$ https://www.icc-cpi.int/CourtRecords/CR2010_02045.PDF>.

75 Situation au Kenya, ICC-01/09-19, Décision relative à la demande d'autorisation d'ouvrir une enquête dans le cadre de la situation en République du Kenya rendue en application de l'article 15 du Statut de Rome (31 mars 2010) (Cour pénale internationale, Chambre préliminaire II), en ligne: CPI $<$ https://www.icc-cpi.int/CourtRecords/CR2011_03256.PDF>.

76 Ibid au para 62.

77 Fernandez et Pacreau, supra note 9 à la p 1195.

78 Ibid.

79 Schabas, « Victor's Justice », supra note 25 à la p 549.

80 Mégret, supra note 63 à la p 429. 


\section{Les chemins de traverse empruntés par les «intérêts de la justice»: une utilisation de l'article 53 au service de l'indépendance du Procureur}

En voulant éviter d'avoir à développer des orientations claires encadrant l'application du critère des intérêts de la justice, le Procureur a mis en place d'autres stratégies (A) qui ont des conséquences sur la transparence de son action (B).

\section{A. «Cherche et trouve»: les prises en considération détournées des « intérêts de la justice »}

La prise en compte des intérêts de la justice se fait à travers celui de la gravité (1) et dans la gestion de la temporalité des examens préliminaires (2).

\section{LES LOIS DE LA GRAVITÉ : LA «GRAVITÉ RELATIVE » COMME COMPOSANTE DES « INTÉRÊTS DE LA JUSTICE »}

Comme le montrent les justifications du Procureur à l'égard du refus d'ouverture d'enquête concernant la situation en $\operatorname{Iraq}^{81}$, son choix est d'appliquer, au sein de l'article 17, le critère de la gravité en premier alors même que celui-ci apparaît secondairement dans la lettre du texte ${ }^{82}$. La jurisprudence a également confirmé cette place première de la gravité dans le processus de l'article $17^{83}$. Ce choix tactique semble être guidé par une impression de facilité quant à l'application de ce critère par rapport à celui de la complémentarité, demandant au Procureur une connaissance détaillée des procédures nationales ${ }^{84}$. Ainsi, comme l'absence d'un des deux critères annule la recevabilité de la situation, le critère de la gravité apparaît comme le plus simple à appliquer en premier. Cette application en vue d'une meilleure effectivité du travail ne pose pas de problème relatif à l'impartialité du Procureur, si ce n'est qu'elle participe à la construction de l'image d'une action fondée essentiellement sur le critère de la gravité.

C'est une autre utilisation du critère de gravité qui doit être questionnée. Ainsi, Stegmiller analyse la redondance du critère de gravité au sein de l'article 53 non comme une répétition formelle, mais comme l'existence de deux types de gravité : une de type juridique qu'il nomme «legal gravity ${ }^{85}$ » et une qu'il nomme «relative gravity ${ }^{86}$ » au sein de laquelle s'applique tout le pouvoir discrétionnaire du

81 Bensadou, supra note 67.

82 Statut de Rome, supra note 1, art 17 (les alinéas (a), (b) et (c) sont consacrés à la complémentarité et l'alinéa (d) fait référence à la gravité).

83 Lubanga Dyilo, supra note 71 au para 29.

84 Ignaz Stegmiller, « The Gravity Threshold Under the ICC Statute: Gravity Back and Forth in Lubanga and Ntaganda » (2009) 9:3 Intl Crim L Rev 547 à la p 548 [Stegmiller].

85 Statut de Rome, supra note 1, art 17, 53(1)(b).

86 Ibid, art 53(1)(c). 
Procureur. Ainsi, l'évaluation de l'impact des crimes mentionnée dans le développement précédent devrait être analysée sous l'article 53(1)(c) en ce qu'il constitue un espace pour la mise en œuvre d'une politique de poursuites voulues par le Procureur. Il utilise la gravité au moment de l'ouverture des enquêtes pour prioriser de potentielles affaires sur d'autres. Il est nécessaire de rappeler ici que les affaires évoquées au moment des examens préliminaires sont entendues au sens large comme les «éventuelles affaires qui pourraient être identifiées au cours de l'examen préliminaire en fonction des renseignements disponibles et qui pourraient résulter de son enquête dans une situation donnée » tel que le décrit le Document de politique générale relatif aux examens préliminaires ${ }^{87}$. L'impact de la gravité des crimes est décrit comme pouvant «s'apprécier à la lumière, entre autres, des souffrances endurées par les victimes et de leur vulnérabilité accrue, de la terreur répandue parmi la population ou des ravages causés sur le plan social, économique et écologique au sein des communautés concernées $^{88}{ }^{\wedge}$ [nos soulignements].

Pour Stegmiller, la notion d'impact renvoie à deux enjeux : un enjeu juridique lié aux souffrances des victimes qui doit être classé dans l'évaluation stricte de la gravité et un enjeu plus large qu'est celui de l'impact des crimes sur les communautés qui doit être renvoyé dans les considérations de l'article 53(1)(c) ${ }^{89}$.

Durant les premières applications du critère de gravité, comme nous l'avons vu pour la situation en Iraq, le Procureur semblait avoir surtout mis en valeur l'aspect numérique des victimes et donc développé une vision quantitative de la gravité. Or, dans sa comparaison avec les autres situations, il mettait en avant une vision plus large du critère et s'aventurait même à évoquer l'impact des crimes puisqu'il dissertait sur le nombre potentiel de personnes déplacées en RDC, en Ouganda et au Soudan $^{90}$, alors qu'il n'adressait pas l'impact des crimes commis par les soldats britanniques sur la population en Iraq. Or, l'application du critère de gravité ne peut, si l'on s'en tient aux valeurs d'objectivité et d'impartialité prônées par le Procureur, se voir appliquer au choix dans certaines situations et pas d'autres ou encore dans un spectre différent : prise en compte des paramètres quantitatif, qualitatif ou les deux.

On notera une redondance de ce problème avec la décision de ne pas ouvrir d'enquête concernant la situation des Comores. L'Union des Comores avait renvoyé une situation concernant le bombardement d'une flottille humanitaire se dirigeant vers la bande de Gaza par un raid israélien au Procureur en mai 2013 ${ }^{91}$. La Procureure reconnaît que des «crimes de guerre relevant de la compétence de la [Cour] avaient été commis sur l'un des navires en cause », mais insiste sur le fait que « le critère de gravité est un critère juridique bien défini par le Statut de Rome » (ce qui est discutable comme nous l'avons vu précédemment). Elle indique par ailleurs qu'elle

\footnotetext{
87 Bureau du Procureur, Document de politique générale relatif aux examens préliminaires, supra note 29 à la $\mathrm{p} 11$.

$88 \quad$ Ibid à la p 17.

89 Stegmiller, supra note 84 à la p 561.

90 Bensadou, supra note 67 à la p 9.

91 Bureau du Procureur, Gaza Freedom Flotilla Situation, supra note 68.
} 
ne minimise pas «l'impact de ces crimes sur les victimes et sur leurs familles », mais qu'elle doit se concentrer sur « les crimes de guerre commis à grande échelle ou dans la poursuite d'un plan ou d'une politique ${ }^{92} »$. Pour la première fois, la Chambre préliminaire I, saisie par l'Union des Comores sur la base de l'article 53(3)(a), a demandé à la Procureure dans sa décision du 16 juillet $2015^{93}$ de reconsidérer sa décision, car même si elle a pris en compte les bons facteurs pour déterminer la gravité, elle n'en a pas fait une bonne application ${ }^{94}$. Par ailleurs, la Chambre repousse en l'espèce la possibilité de l'expression d'un pouvoir discrétionnaire dans l'évaluation du critère de gravité. Ce point sera développé dans la partie B.

\section{LA PRISE EN COMPTE DE LA TEMPORALITÉ DANS LES EXAMENS PRÉLIMINAIRES}

Deux aspects seront abordés ici : la longueur des examens préliminaire et le choix du séquençage des enquêtes. Une des stratégies visant à ne pas refuser l'ouverture d'une enquête sur les bases des intérêts de la justice consiste tout simplement à ne pas terminer l'examen préliminaire, reportant ainsi toute justification des résultats de l'examen préliminaire. Ainsi, selon les procédures de contrôle prévues par l'article 53, que nous verrons en détail dans le développement suivant, pour qu'il puisse y avoir un contrôle de la Chambre, il faut que celui-ci se fasse suite à la décision du Procureur fondée sur les critères de l'article 53 et à la notification de celle-ci. Cet agencement procédural a pour conséquence l'absence de tout contrôle en l'absence de décision. Ce vide a été décelé dans la situation relative à la République centrafricaine. En l'occurrence, la situation avait été référée par le gouvernement centrafricain au Procureur le 22 décembre 2004 et aucune indication de l'avancement n'avait été donnée par le Procureur en 2006, date à laquelle la Chambre préliminaire III a rendu sa décision relative à la demande d'informations sur l'état d'avancement de l'examen préliminaire ${ }^{95}$. Dans celle-ci, la Chambre préliminaire indique que «quelle que soit sa complexité », l'examen préliminaire doit être achevé dans un délai raisonnable ${ }^{96}$ et demande ainsi au Procureur de lui communiquer un rapport de l'état d'avancement de l'examen ${ }^{97}$. Pour El Zeidy, ce vide donne carte

92 Bureau du Procureur, Déclaration du Procureur de la Cour pénale internationale Fatou Bensouda à propos de la cloture de l'examen préliminaire relatif à la situation renvoyée par les Comores (novembre 2014), en ligne : CPI <https://www.icc-cpi.int/Pages/item.aspx?name=otp-statement-06-11$2014 \& \ln =\mathrm{fr}>$ [Bureau du Procureur, Fatou Bensadou].

93 Situation renvoyée par l'Union des Comores, ICC-01/13-34, Decision on the Request of the Union of the Comoros to Review the Prosecutor's Decision not to Initiate an Investigation (16 juillet 2015) (Cour pénale internationale, Chambre préliminaire I), en ligne: CPI <https:/www.icc-cpi.int/ CourtRecords/CR2015_13139.PDF> [Situation renvoyée par l'Union des Comores].

$94 \quad$ Ibid à la p 11.

95 Situation en République centrafricaine, ICC-01/05-Tfra, Décision relative à la demande d'information sur l'état d'avancement de l'examen préliminaire de la situation en République centrafricaine (30 novembre 2006) (Cour pénale internationale, Chambre préliminaire III), en ligne : CPI $<$ https://www.icc-cpi.int/CourtRecords/CR2007 04256.PDF>.

96 Ibid à la $\mathrm{p} 4$.

$97 \quad$ Ibid à la p 5. 
blanche au Procureur et il estime que cette situation entre dans le spectre de l'aphorisme «justice delayed, justice denied $»^{98}$. Pour Fernandez et Pacreau, « ce qui devait être préliminaire se transforme en une sorte de contrôle continu des procédures nationales existantes et devient plutôt un instrument de pression entre les mains du Procureur» et cette technique constituerait donc un encouragement à la complémentarité positive ${ }^{99}$. Cette configuration peut être observée concernant la situation en Colombie, qui fait l'objet d'un examen préliminaire depuis 2004, permettant ainsi au Procureur de garder une marge de manœuvre tout en observant la mise en œuvre des actions nationales en matière de justice.

Cette latence nous permet seulement d'émettre des hypothèses. Ainsi, le Procureur a pu penser que le moment n'était pas approprié pour une ouverture d'enquête, mais malheureusement l'absence de justification de sa part ne nous permet pas de connaître les facteurs qu'il prend en compte et qui pourraient nous permettre de mieux comprendre quels sont les paramètres des conditions contraires aux intérêts de la justice et dans quelles mesures, il s'attache au contexte national, hors de la prise en compte stricte de procédures pénales.

De même, comment interpréter les politiques de poursuites qui, depuis Moreno Ocampo et sous Fatou Bensouda également, font la promotion du séquençage des actions du Bureau du Procureur? Ainsi, dans l'échelle de la gravité, certaines affaires prennent le pas sur d'autres dans le cadre de la même situation et par conséquent, certaines enquêtes sont ouvertes avant d'autres. C'est ainsi qu'a été justifié par exemple le choix de faire porter des poursuites en Côte d'Ivoire sur Laurent Gbagbo et le retard relatif à l'enquête portant sur les violences postélectorales commises par le camp Ouattara. En effet, Fatou Bensouda a annoncé que les enquêtes concernant le camp d'Alassan Ouattara n'avaient débuté qu'à la mimai $2015^{100}$, bien après celles relatives à Laurent Gbagbo. Il serait nécessaire dans ce type de configuration de connaître les éléments de gravité sur lesquels se basent le Bureau pour choisir l'ordre de priorisation des enquêtes et savoir si ces éléments entrent dans la catégorie «legal» ou "relative» de la gravité et si ce n'est pas une anticipation des intérêts de la justice tels que formulés dans l'article 53(2)(c) qui comprend l'évaluation du rôle de l'auteur présumé dans le crime allégué.

\section{B. Les conséquences procédurales du refus d'ouvrir une enquête sur le contrôle du pouvoir discrétionnaire du Procureur}

La règle $105 \mathrm{du}$ Règlement de procédure et de preuve de la Cour prévoit que le Procureur doit informer l'État qui a référé la situation ou le Conseil de sécurité s'il

$98 \quad$ El Zeidy, supra note 63 aux pp 54- 55.

99 Fernandez et Pacreau, supra note 9 à la p 1186.

100 Marc Perelman, «Fatou Bensouda sur France 24 : les enquêtes concernant les pro-Outtara débuteront à la mi-mai » Afrique News (3 avril 2015), en ligne : afriquenewsinfo.net <http://afriquenewsinfo.net/ 2015/04/03/fatou-bensouda-sur-france24-les-enquetes-concernant-les-pro-ouattara-debuteront-a-la-mimai-2015/>. 
décide de ne pas ouvrir une enquête ${ }^{101}$. Cette notification doit comprendre les motifs et les conclusions de la décision ${ }^{102}$. Si cette décision se base seulement sur les «intérêts de la justice» («raisons sérieuses de penser»), il doit également en informer la Chambre préliminaire ${ }^{103}$. On notera, au vu du développement précédent, que cette première étape peut déjà être problématique : l'absence de notification a pour conséquence une absence de possibilité d'évaluation par la Chambre préliminaire. L'article 53 prévoit plusieurs types de procédures afin de contrôler les choix effectués par le Procureur d'ouvrir une enquête. L'élément le plus intéressant de ce système de contrôle est la différenciation des mécanismes de contrôle prévus en fonction de la base légale sur laquelle s'appuie le Procureur pour prendre sa décision. Ainsi, l'article 53(3)(a) prévoit que la Chambre préliminaire peut examiner la décision du Procureur et lui demander de la reconsidérer, si celle-ci est basée sur les paragraphes 1 et 2, et que cette demande provient de l'État ou du Conseil de sécurité qui ont respectivement référé la situation. L'article 53(3)(b) prévoit, quant à lui, que la Chambre préliminaire peut proprio motu examiner la décision, si celle-ci est seulement fondée sur le critère des intérêts de la justice au paragraphe 53(1)(c) et 53(2)(c). Cette décision n'aura d'effet «que si elle est confirmée par la Chambre préliminaire $^{104} \gg$ [nos soulignements]. Il est toutefois nécessaire de noter que l'article 53(4) permet au Procureur de reconsidérer sa décision s'il a de nouveaux renseignements. On soulèvera ici le fait que les conséquences soient différentes, pour le même critère, à savoir celui des intérêts de la justice, en fonction de la provenance de la requête ${ }^{105}$.

La conséquence principale d'un tel système est la suivante : le pouvoir discrétionnaire du Procureur ne peut être contredit avec conséquence (à savoir l'obligation pour le Procureur d'ouvrir une enquête imposée par la Chambre) que si son refus se base sur le critère des "intérêts de la justice» et que la Chambre agit proprio motu. Ainsi, si le Procureur utilise son pouvoir discrétionnaire dans le cadre du critère de gravité et qu'il est argumenté que celui-ci provient de l'article 17, qui correspond à l'article 53(1)(b), alors la Chambre préliminaire ne peut que lui demander de reconsidérer sa décision, donc sans obligation de changement. $A$ contrario, concernant les intérêts de la justice, s'il y a refus d'ouverture d'une enquête sur cette base, le Chambre préliminaire peut en théorie obliger le Procureur. Or, comme nous l'avons dit précédemment, aucun refus d'ouverture d'enquête n'a jamais été soutenu par le Procureur sur la base des intérêts de la justice alors que cela a été fait sous la coupe du critère de gravité. Schabas met en lumière le fait qu'il est étrange que les conséquences soient différentes pour les intérêts de la justice en fonction de

101 Règlement de procédure et de preuve, 3-10 septembre 2002, ICC-ASP/1/3 (Part II-A), en ligne : CPI $<$ https://www.icc-cpi.int/iccdocs/PIDS/legal-texts/RulesProcedureEvidenceFra.pdf $>$ [Règlement de procédure et de preuve].

102 Ibid, règle $105(3)$.

103 Ibid, règles 105(4) et 105(5).

104 Statut de Rome, supra note 1, art 53(3)(b).

105 Schabas, International Criminal Court, supra note 23 ( [i]t seems strange that the consequences are different depending upon the source of the request for the review, but this is what both the Rome Statute and the Rules of Procedure and Evidence indicate » à la p 669). 
qui demande la révision ${ }^{106}$. Pour Heller, " [l] imiting review to declinations based on the 'interests of justice' factor thus makes little sense, especially given that the OTP has specifically disclaimed the idea that the factor permits it to consider the political impact of its investigations ${ }^{107} »$.

Pour Turone, il faudrait que la gravité soit évaluée dans le cadre des intérêts de la justice ${ }^{108}$. Il rejoint donc la position de Stegmiller, pour qui la Chambre préliminaire devrait pouvoir évaluer ce critère qui laisse place au pouvoir discrétionnaire et renverser la décision du Procureur si nécessaire ${ }^{109}$. Dans le Statut, c'est donc au Conseil de sécurité qu'il revient de suspendre une enquête si celle-ci entrave les nécessités de paix. Or, nombre d'auteurs sont sceptiques sur cette capacité. Ainsi, Rodman indique que « [w] hile the Security Council is a political institution, its resolutions often reflect a least-common-denominator compromise of national interests rather than a coherent political strategy ${ }^{110} »$. Schabas, quant à lui, met de l'avant le fait que l'article 16 a été simplement inséré dans le Statut comme une "concession to buy the acquiescence of the permanent members of the Security Council. It was never intended to provide a scheme for the exercise of political discretion where a situation or a case is otherwise admissible ${ }^{111} »$.

L'articulation de ces contrôles et surtout les interstices qu'ils laissent à l'action du Procureur ne sont pas dommageables en ce qu'ils permettent au pouvoir discrétionnaire de s'exercer - cela est nécessaire pour préserver son indépendance -, mais en ce qu'ils ont pour conséquence de ne jamais éclairer et donc ouvrir le débat sur les considérations prises en compte par ce dernier, ternissant par conséquent la légitimité de son action ${ }^{112}$. Ainsi pour Heller, un contrôle de la Chambre préliminaire aurait pu avoir une conséquence concernant la situation en Iraq, puisqu'il indique que le Procureur sera toujours plus enclin à ouvrir une enquête concernant une situation où il a des informations indiquant un très grand nombre de victimes, plutôt qu'une situation où il $\mathrm{y}$ a peu de victimes, mais pour laquelle il faudrait une enquête pour conclure à la systématicité ou non des crimes, comme cela est le cas pour les crimes de guerre commis par les Britanniques ${ }^{113}$. Cela empêche donc une évaluation plus approfondie de l'aspect qualitatif de la gravité (circonstances des crimes).

Cette impasse va-t-elle changer suite à la décision relative à la flottille ? Dans le cas de la situation référée par les Comores, notifiant que le Procureur n'avait pas

\footnotetext{
106 Ibid.

107 Kevin J Heller, « Situational Gravity Under the Rome Statute » dans Carsten Stahn et Larissa Van den Herik, dir, Future Perspectives on International Criminal Justice, La Haye, TMC Asser, 2010, 227 à la p 250 [Heller].

108 Turone, supra note 11 à la p 1156.

109 Stegmiller, supra note 84.

110 Kenneth A Rodman, « Is Peace in the Interests of Justice? The Case for Broad Prosecutorial Discretion at the International Criminal Court » (2009) 22:1 Leiden J Intl L 99 à la p 122 [Rodman].

111 Schabas, supra note 25 à la p 551.

112 Carsten Stahn et Göran Sluiter, dir, The Emerging Practice of the International Criminal Court, Boston, Boston, Martinus Nijhoff, 2009 à la p 279.

113 Heller, supra note 107 à la p 34.
} 
fait une bonne évaluation du critère de gravité, la Chambre préliminaire indique qu'elle «recognises that the Prosecutor has discretion to open an investigation but, as mandated by article 53(1) of the Statute, that discretion expresses itself only in paragraph (c) [...] Conversely, paragraphs (a) and (b) require the application of exacting legal requirements ${ }^{114} \gg$.

Elle exclut donc en l'espèce l'utilisation du critère de gravité comme requérant le pouvoir discrétionnaire du Procureur - affirmation contestée par le juge Kovacs dans son opinion dissidente ${ }^{115}$ - et pousse par conséquent le Procureur à justifier sa décision sous les «intérêts de la justice». Comme on l'a déjà fait remarquer à plusieurs reprises, la conceptualisation du critère de gravité reste problématique à la $\mathrm{CPI}^{116}$ (nécessite-t-elle la prise en compte de l'impact social ? Estelle un centre d'expression du pouvoir discrétionnaire du Procureur?) et cette décision indique que sous l'article 17, la gravité ne doit pas inclure des choix de politique de poursuite, mais des critères objectifs de gravité. On peut ici noter une contradiction dans le raisonnement : comment la notion d'impact des crimes prise en compte en l'espèce peut-elle faire l'objet d'une évaluation objective ?

Cela nous renvoie à la question: le Procureur doit-il faire évoluer son interprétation des «intérêts de la justice »? Nous pensons que oui, mais à certaines conditions.

\section{Une nécessaire évolution du spectre du critère des intérêts de la justice : Quand la trop grande indépendance nuit à la légitimité...?}

La posture légaliste du Procureur au sein du débat paix/justice, à savoir qu'il ne doit pas prendre en considération l'aspect politique de la paix du fait de sa mission purement judiciaire, ne semble pas pouvoir résister à la réalité des terrains d'action, soit des conflits en cours ou des situations en phase de stabilisation. Qu'en est-il quand l'action du Procureur peut être dommageable à la recherche de la paix ? La possibilité de prise en compte de ces considérations dans les «intérêts de la justice» va être évaluée (A) ainsi que la possibilité d'une grille de lecture permettant sa mise en œuvre (B).

114 Situation renvoyée par l'Union des Comores, supra note 93 à la p 8.

115 Situation renvoyée par l'Union des Comores, ICC-01/13-34, Partly Dissenting Opinion of Judge Peter Kovacs, ICC-01/13-34-Anx (Cour pénale internationale, Chambre préliminaire I) (16 juillet 2015), en ligne : CPI <https://www.icc-cpi.int/RelatedRecords/CR2015_13151.PDF>.

116 Pour une analyse détaillée et critique des défauts de l'application de la gravité par la Chambre préliminaire, voir Dov Jacobs, «ICC Judges Ask the Prosecutor to Reconsider Decision not to Investigate Israeli Gaza Flotilla Conduct» (juillet 2015), en ligne: Spreading the Jam $<$ dovjacobs.com/2015/07/20/icc-judges-ask-the-prosecutor-to-reconsider-decision-not-to-investigateisraeli-gaza-flotilla-conduct/>; Kevin J Heller, « The Pre-Trial Chamber's Dangerous Comoros Review Decision » (juillet 2015), en ligne : Opinio Juris <opiniojuris.org/2015/07/17/the-pre-trial-chambersproblematic-comoros-review-decision/>. 


\section{A. Pour une interprétation différente du critère des intérêts de la justice : arguments et limites}

La doctrine s'est beaucoup interrogée sur les impacts d'une ouverture d'enquête dans le cas où le conflit serait encore en cours ou lorsque des négociations d'accords de paix seraient en train d'avoir lieu.

Rodman met en lumière la difficulté de position d'un Procureur qui ferait l'annonce d'une enquête ayant pour conséquence la reprise des hostilités, la violation d'un cessez-le-feu ou l'augmentation du degré de violence du conflit. En effet, cela aurait pour conséquence que les acteurs politiques, sur lesquels la coopération repose, soient moins enclins à travailler avec lui. Pour l'auteur, il est alors nécessaire de distinguer un Procureur sous influence politique et un Procureur conscient de l'environnement dans lequel son travail aura lieu et qui prend conseil auprès d'autres organisations, comme les Nations unies ou des ONG concernant l'impact d'une enquête $^{117}$. Ludwin, quant à elle, explique avec détails en quoi la réponse d'une justice transitionnelle composée de façon monolithique par le procès pénal ignore toutes les nuances et les complexités d'un conflit ${ }^{118}$. Bluemenson ancre sa volonté d'une interprétation large des intérêts de la justice dans le respect de la diversité et de la pluralité, mettant en lumière que le défi est de définir «the proper scope and necessary limits of diversity in state approaches to accountability. No one has been able to precisely locate the points at which legitimate moral diversity ends and universal moral imperatives begin, but it is a central issue of our time, and an unavoidable one for the Court ${ }^{119}$ ».

Il apparaît ici indispensable de parvenir à définir comment les diversités de réponses à un conflit doivent être prises en compte par le Procureur. C'est une composante particulière de la justice transitionnelle qui est problématique : les CVR et les amnisties qui peuvent en résulter. Ces processus doivent-ils être considérés sous l'article 17 en tant que complémentarité, ou sous l'article 53(1)(c) comme participant à la considération des " intérêts de la justice » ? Robinson rappelle les deux principaux enjeux des discussions lors des travaux préparatoires autour de l'article 17 (les débats portaient autour de deux problématiques : quelle justice est la plus appropriée à la sortie d'un conflit? et Introduire une exception dans l'article 17 va-t-il à l'encontre du Statut ?), explicitant par là même le choix des rédacteurs du Statut de ne pas inclure explicitement une provision sur les amnisties dans l'article 17 par peur de la formulation d'une exception permettant les dérives des comportements étatiques ${ }^{120}$. Hethe Clark parvient à la même conclusion en indiquant que :

117 Rodman, supra note 110 à la p 121.

118 Elizabeth L King, « Does Justice Always Require Prosecution? The International Criminal Court and transitional justice measures » (2013) 45:1 Geo Wash Intl L Rev 85 à la p 93.

119 Eric Bluemenson, « The Challenge of a Global Standard of Justice: Peace, Pluralism, and Punishment at the International Criminal Court » (2006) 44:3 Colum J Transnatl L 801 à la p 872.

120 Darryl Robinson, «Serving the Interests of Justice: Amnesties, Truth Commissions and the International Criminal Court » (2003) 14:3 Eur J Intl L 481 à la p 483 [Robinson]. 
[t] he Rome Statute's drafters understood that the use of these programs may, at times, be an unfortunate necessity and that the argument, when "stripped to its essence ... is one of lesser evils." Because of these considerations, the legal regime established by the Rome Statute does not, in cases where the jurisdictional requirements of the Court are otherwise met, foreclose the use of amnesties and alternative justice mechanisms when they are in "the interests of justice"121.

Cette exclusion des CVR et des amnisties de l'article 17 est aussi défendue par Allan qui explique que les alinéas (a) et (b) de l'article 17(1) doivent être lus ensemble rendant interdépendantes les obligations d'enquêtes, de poursuites et de réparation ${ }^{122}$. Dans ce contexte, les CVR ne pourraient pas répondre à toutes les caractéristiques voulues par l'article 17 pour écarter l'ouverture d'une enquête.

Si les développements précédents semblent nous porter vers une prise en compte d'une justice restorative à travers les intérêts de la justice, cela n'est pourtant pas sans obstacle. En effet, l'exercice du pouvoir discrétionnaire sous l'article 53(1)(c) ne peut tout autoriser. Certaines questions restent toujours en suspens : qui doit être concerné par ces mesures? Quels sont les critères que le Procureur doit prendre en compte?

Plusieurs auteurs ont plaidé pour plus de transparence. McDonald et Haveman expliquent que les choix effectués à des stades aussi précoces que l'ouverture d'enquête vont influencer la perception qu'a le public du travail de la Cour sur le long terme et que donc "[I]t is therefore of critical importance that the OTP [Bureau, NDLR] gives long and hard thought to the issue of prosecutorial discretion and how it should be exercised ${ }^{123} »$. Dans la même perspective, pour Danner, c'est parce que le Procureur participe à la construction de l'image de légitimité de la Cour qu'il doit faire preuve de transparence dans ses décisions ${ }^{124}$. Ce paramètre semble avoir été pris en considération, de manière générale, avec l'arrivée de Fatou Bensouda à la Cour. En effet, cette dernière a multiplié la publication de documents de politique générale (crimes sexuels et à caractère sexiste, politique à l'égard des enfants, sélection et hiérarchisation des affaires). Ceux-ci à forte valeur pédagogique ont pour but de rendre moins obscur le travail du Bureau et de démontrer la pertinence de ses actions. Ainsi, cette volonté de prouver que la CPI reste une actrice pertinente dans les grandes crises pour lesquelles elle a compétence couplée au tournant pédagogique pris sous le mandat de Fatou Bensouda semblent créer des conditions favorables faisant espérer une plus grande transparence dans l'analyse faite par le Bureau du critère des intérêts de la justice.

121 Clark, supra note 53 à la p 407.

122 Allan, supra note 53 aux pp 251- 52.

123 Avril McDonald et Roelof Haveman, Prosecutorial Discretion - Some Thoughts on "Objectifying” the Exercise of Prosecutorial Discretion by the Prosecutor of the ICC, La Haye, Expert Consultation Process on General Issues Relevant to the ICC Office of the Prosecutor, 2003 aux pp 2- 3.

124 Danner, supra note 4 à la p 511. 
Dans le développement suivant, nous allons discuter des considérations à prendre en compte dans l'évaluation des «intérêts de la justice», mais avant, nous souhaitons éclairer toute la difficulté de mettre en place de telles considérations en soulevant les interrogations mises en lumière par Greenawalt ${ }^{125}$. Ainsi, l'auteur se questionne sur la capacité de critères à s'adapter à la complexité et à la diversité des situations rencontrées ${ }^{126}$ et sur les conséquences d'une consécration juridique (dans le cas où les Chambres préliminaires contrôlent le raisonnement du Procureur sur la base de l'article 53(1)(c) de problématiques d'ordre politique, que sont par exemple l'octroi d'amnisties ${ }^{127}$. Dans la même perspective relative à la capacité d'étreinte des réalités politiques par le droit, Allan se demande comment adresser la différence pouvant exister entre la fonction de jure et de facto d'un individu dans un conflit armé et donc l'application de potentiels critères ${ }^{128}$.

Malgré ces réticences, nous pensons que ces derniers peuvent être profitables à la construction de la légitimité de l'action du Procureur en expliquant ces choix, tout en gardant en tête les mises en garde précédentes rappelant l'indispensable analyse au cas par cas des situations.

\section{B. Proposition d'une grille de lecture encadrant l'interprétation des intérêts « des justices »}

S'interroger sur l'application des critères revient à s'interroger sur la manière dont le Procureur use de son pouvoir discrétionnaire. Or, celui-ci n'obéit à aucune grille de lecture précise. C'est pourquoi beaucoup d'auteurs ont tenté d'en conceptualiser une afin de rendre le processus plus transparent. Nous faisons référence ici à ce que Philippa Webbnomme les «ex ante criteria ${ }^{129}$. Nous avons choisi de mettre en lumière ici les critères qui nous semblaient les plus pertinents, toujours dans l'objectif de faire gagner en légitimité l'action du Procureur.

Tout d'abord, cette interprétation plus extensive du critère des intérêts de la justice ne signifie pas une multiplication des refus d'ouverture d'enquête, ni la marque d'une position idéologique refusant les poursuites pénales à la sortie d'un conflit. Ainsi, la règle fondamentale reste la même: les plus hauts responsables doivent être poursuivis pour les crimes commis et l'exception à cette règle doit rester extrêmement encadrée. De même, la charge des poursuites incombe en premier lieu aux États. Ainsi, Hethe Clark rappelle la contradiction qui ressortirait pour le crime de génocide et les infractions graves aux lois et coutumes de la guerre de la prise de décision de ne pas poursuivre alors même que les règles du droit international

125 Alexander K A Greenawalt, « Justice Without Politics? Prosecutorial Discretion and the International Criminal Court » (printemps 2007) 39:3 NYU J Int'l L \& Pol 583 [Greenawalt].

126 Ibid à la p 656.

127 Ibid aux pp 659-660.

128 Allan, supra note 53 à la p 297.

129 Philippa Webb, « The ICC Prosecutor's Discretion not to Proceed in the "Interests of Justice” » (2005) 50:3 Crim LQ 305 [Webb]. 
indiquent cette obligation (la situation étant plus délicate pour les crimes contre l'humanité en l'absence de dispositions en ce sens $)^{130}$. Ainsi, il paraîtrait contradictoire pour la justice internationale pénale, attachée aux symboles, de ne pas poursuivre là où les États s'y voient contraints par des règles internationales.

Webb offre un panorama plus large des considérations à prendre en compte, en plus de celles listées dans l'article 53 : les ressources de la Cour (considéré comme le critère le moins décisif), la paix et la sécurité internationales, l'ensemble des aspects de la justice transitionnelle, des considérations transversales (principe de nondiscrimination, effet dissuasif et la perception de l'intégrité du système judiciaire) ${ }^{131}$. Nous pensons que ces critères sont interdépendants et que l'affirmation de critères prenant en compte la paix et la sécurité ainsi que la justice transitionnelle aura des impacts sur les considérations transversales dont elle parle.

La formulation de paramètres concrets au moment de l'ouverture d'une enquête renforcerait la position du Procureur en tant qu'acteur de premier plan de la justice transitionnelle de même que cela éclairerait sa capacité à jouer en réseau avec les autres acteurs clés de la transition. Cela participerait à la fois à la déconstruction de la perception selon laquelle la justice transitionnelle constituerait une tour de Babel où règne l'incompréhension entre acteurs et d'autre part cela renforcerait la légitimité des actions du Bureau. En effet, nous pensons que celle-ci se nourrit tout autant par les actes que par leur absence.

La grille de lecture propice à une interprétation plus large des intérêts de la justice concerne deux phases du conflit : la phase de transition qui prend en compte les CVR et les amnisties et la phase où le conflit est toujours en cours.

Concernant la transition, des critères sont donnés par Goldstone et Fritz sur les caractéristiques des CVR dont résultent les amnisties : elles doivent être dotées des ressources nécessaires afin de mener des enquêtes, être le résultat d'un processus démocratique, les coupables doivent voir leur responsabilité reconnue publiquement et les victimes avoir une véritable place dans le processus (publicité et diffusion des informations récoltées et indépendance de l'entité du gouvernement $)^{132}$. Robinson fait ainsi trois propositions afin de guider l'interprétation des « intérêts de la justice » au regard des amnisties qui pourraient être accordées. La première que nous avons déjà affirmée est que la règle de base est celle de la poursuite ${ }^{133}$. La deuxième dispose que dans les situations de transition, un programme basé sur la poursuite seulement des plus hauts responsables peut être considéré comme acceptable ${ }^{134}$. La troisième est une situation qualifiée par l'auteur de «drastic neccesity», dans laquelle les amnisties

30 Clark, supra note 53 aux pp 398- 99.

131 Webb, supra note 129.

132 Richard Goldstone et Nicole Fritz, «"In the Interests of Justice” and Independent Referral: The ICC Prosecutor's Unprecedented Powers » (2000) 13:3 Leiden J Intl L 655.

133 Robinson, supra note 120 à la p 488.

134 Ibid à la p 493. 
seraient accordées à tous les auteurs («blanket amnisties $»){ }^{135}$. Pour l'auteur, la notion de «nécessité » des amnisties pour qu'une situation s'améliore doit être considérée de façon rigoureuse et ne doit être utilisée que de façon exceptionnelle en évaluant minutieusement les menaces à la reprise de la violence ${ }^{136}$. Il justifie ce positionnement en s'appuyant sur des études remettant en question l'effet pacificateur des amnisties ${ }^{137}$ et propose quelques considérations à prendre en compte avant toute acceptation (qualité de l'enquête, indépendance et ressources à disposition, l'absence de poursuites de tous les responsables sont-elles basées sur des réalités sociales, économiques et politiques qui rendent tout autre choix impossible, la publicité des noms des responsables, les formes de punition des coupables, réparation pour les victimes, volonté de prendre en compte les droits humains). Nous pensons que l'ensemble de ces critères permet au Procureur de prendre en compte la diversité des environnements auxquels il est confronté sans pour autant laisser "carte blanche» à l'impunité. La reconnaissance d'une action impartiale et légitime du Procureur dans son refus d'ouvrir une enquête provient majoritairement de la prise en compte du caractère démocratique des mesures mises en place sur le territoire national.

Qu'en est-il quand aucune de ces mesures n'a encore été mise en place? Pour Greenawalt, il est nécessaire que les enquêtes soient acceptées, et ce, de manière systématique, dans les conflits en cours. En effet pour l'auteur, les enquêtes sont préférables à la supposition d'une quelconque forme de justice ${ }^{138}$. Nous sommes toutefois en désaccord avec sa vision d'enquêtes et de poursuites qui doivent porter selon lui sur les "unambigous evils that are universally recognizable as such ${ }^{139}$ » et qui supposent l'intégration par le Procureur d'une vision préconçue d'un conflit transmise par les acteurs politiques les plus influents de la scène internationale. En effet, selon nous, les enquêtes dans cette configuration précise doivent concerner toutes les parties et la sélection doit se faire plus tard dans le cadre de la sélection des poursuites telle que décrite à l'article 53(2).

En conclusion, le critère de pondération qu'est celui des intérêts de la justice dans le cadre de l'ouverture des enquêtes, tel que formulé dans l'article 53(1)(c), a été interprété de manière restrictive par le Procureur qui a affirmé son application exceptionnelle, dans la perspective d'une présomption favorable aux poursuites et donc à l'ouverture des enquêtes. Cette vision, portée par une conception de la justice transitionnelle comme reposant fondamentalement sur la justice rétributive, a eu pour conséquence l'absence de son invocation par le Procureur dans le choix du refus de l'ouverture de ses enquêtes jusqu'ici.

En apparence donc, le critère des intérêts de la justice, que le Procureur se doit d'expliciter seulement quand l'ouverture d'une enquête irait à son encontre, ne guide pas son choix puisque les enquêtes iraient toujours dans le sens des intérêts de

Ibid à la p 495.

Ibid.

Ibid à la p 496.

138 Greenawalt, supra note 125 à la p 663.

139 Ibid. 
la justice. Dans la pratique, le pouvoir discrétionnaire du Procureur s'exerce à travers l'application du critère de la gravité, qui fait l'objet d'une appréciation quantitative et qualitative et qui a été invoqué à plusieurs reprises par le Procureur pour justifier la non-nécessité d'une enquête. Une partie de l'évaluation de ce critère (comme la notion d'impact par exemple) est considérée par certains auteurs comme pouvant entrer dans l'application de l'article 53(1)(c) car cette disposition est perçue comme la seule autorisant l'exercice d'un pouvoir discrétionnaire. On peut également considérer que la pratique du Procureur concernant le délai des examens préliminaires et son choix de prioriser des enquêtes sur d'autres comme un exercice de son pouvoir discrétionnaire pouvant entrer sous l'article 53(1)(c). Ces deux comportements peuvent être interprétés comme une manière pour le Procureur de prendre en compte le critère des intérêts de la justice sans avoir à clairement le formuler. Ces utilisations détournées ont des conséquences sur la manière dont les refus d'enquête ont été évalués par les Chambres préliminaires. Cela risque néanmoins de changer avec la décision de la Chambre préliminaire sur la situation aux Comores, qui a pour conséquence de pousser le Procureur à se justifier sous l'article 53(1)(c).

Afin de participer à la légitimité des actions du Procureur, nous pensons que ce dernier a intérêt à élargir et clarifier sa prise en compte des considérations relatives aux intérêts de la justice, notamment dans les situations où une transition ou un conflit sont en cours, afin de pallier aux considérations lacunaires contenues dans le document relatif aux intérêts de la justice, qui ne reflètent pas la réalité de son action. Cette nécessité d'explication des actions du Bureau semble comprise par Fatou Bensouda qui multiplie les documents expliquant l'action de son Bureau, malgré des lacunes persistantes quant aux intérêts de la justice. De cette considération résulte la prise en considération d'un paramètre non juridique dans l'analyse de l'exercice du pouvoir discrétionnaire du Procureur, celui de la volonté personnelle de l'individu à ce poste d'éclairer son action. 